\title{
Inactivation of Bacterial Spore, Endotoxin, Lipid A, and Abnormal Prion by Gas Plasma Exposure
}

\section{Hideharu Shintani*}

Faculty of Science and Engineering, Chuo University, Tokyo, Japan

The infections can be attributed to various microorganisms such as Staphylococcus aureus, E. coli, Bacillus cereus and so on which can be present on the surface of healthcare products as bioburden and can come into contact with human tissues. Several studies have demonstrated that some of these microorganisms are resistant to antibiotics such as MRSA, VRE and so on and have an impact on the patients; this indicates the need for improved sterilization procedures free from bioburden, airborne, falling microorganisms or adhesive microorganisms as far as possible. However, some studies have shown that significant quantities of residues composed of salts, proteins and organic matter are left on operational instruments even after complete cleaning and sterilization. These observations raise serious health concerns because residue pathogens can be transmitted to pathogen free patients as iatrogenic diseases. Most serious pathogens included are the abnormal prion proteins, the principal factors for the transmission of Creutzfeld Jakob Disease; this could potentially be transmitted with contaminated instruments during brain operation.

Another concern is related to pyrogens (fever-inducing substances) deriving from gram-negative bacteria (1ipopolysaccharides (LPS)), endotoxin or Lipid A (Figures 1). These LPS, bacterial endotoxins or lipid A, can cause fever when in contact with the bloodstream and provoke fever shock, which is a major cause of death among, for example, dialysis patients. Endotoxins are extremely resistant to temperature and are not removed by conventional sterilization procedures such as autoclave sterilization or $\gamma$-ray or e-beam sterilization. A few papers were published on the effect of endotoxin by gas plasma exposure at more than $5 \log$ reduction during $30 \mathrm{~min}$ exposure by nitrogen gas plasma. In general $3 \log$ reductions were required by authorities and GMP (good manufacturing practice) and according to Figure 1, during $10 \mathrm{~min}$ at $28-45^{\circ} \mathrm{C}$ operation, $3 \log$ reduction were completed, which is significant achievement.

Sterilization procedures must follow official rules such as ISO 14937 and 14161 and so on. The normal procedure consists of treatments in mechanical washing/disinfection medical devices: they are, first, prewashed to prevent blood coagulation and adhesion of protein. The instruments are then cleaned and sonicated with an alkaline enzymatic detergent and, finally, washed at high temperature and rinsed. They are then visually inspected after drying and packaged for sterilization, which in most cases can be carried out by autoclave sterilization, which is inferior procedure of inactivation of endotoxin and abnormal prion proteins. Because these operations are not efficient in completely removing organic residues, and other sterilization methods are also unable to destroy endotoxin and abnormal prions completely. However, Shintani et al succeeded in inactivation of endotoxin and prions by nitrogen gas plasma exposure [1-3]. Moreover, the usual sterilization techniques have several drawbacks including the potential degradation of heat-sensitive healthcare products (e.g., autoclave sterilization at $121.11^{\circ} \mathrm{C}$ for $20 \mathrm{~min}$ in normal); relatively long operation time due to toxic gas degassing (ethylene oxide gas or hydrogen peroxide gas sterilization). The autoclave sterilization is required $121.11^{\circ} \mathrm{C}$ for 20 min, $\gamma$-ray sterilization of $25 \mathrm{kGy}$ is $2.5 \mathrm{~h}(10 \mathrm{kGy} / \mathrm{h}$ in normal) and electron-beam sterilization is a few $\mathrm{s}$, therefore sterilization procedures are not always required too long process times in every cases. It depends on sterilization procedures selected.

Alternative sterilization procedures are under investigation including the use of non-equilibrium gas plasma discharges: we will see that plasma processes are able to sterilize bacterial spore with more than $6 \log$ reduction without residue of toxic gases (Figure 2) [4] and conducted at relatively low temperature at $25-60^{\circ} \mathrm{C}$ for endotoxin inactivation for more than $5 \mathrm{log}$ reduction [1-4]. Non-equilibrium gas plasma sterilization was carried out to show that many pathogens can be inactivated $[1,2]$ and mechanism and inactivation factors of gas

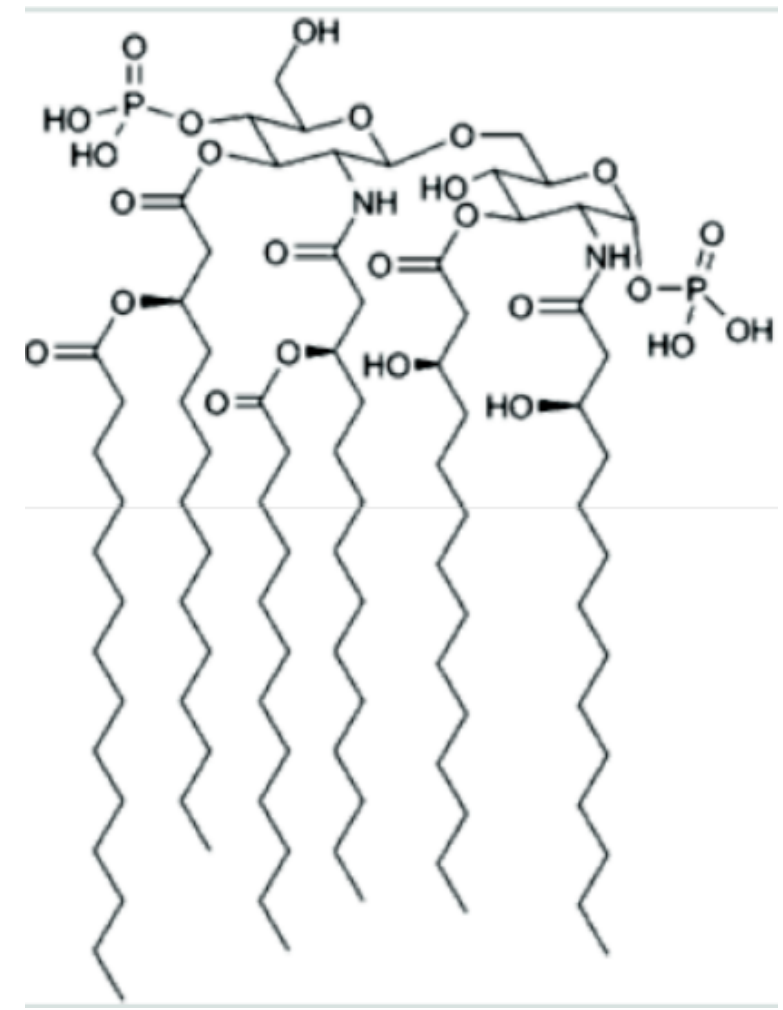

Figure 1: Chemical structure of Lipid A.

*Corresponding author: Hideharu Shintani, Faculty of Science and Engineering, Chuo University, 1-13-27, Kasuga, Bunkyo, 112-8551, Tokyo, Japan, Tel: +81425922336, Fax: +81425922336, E-mail: shintani@mail.hinocatv.ne.jp

Received March 07, 2015; Accepted March 09, 2015; Published April 13, 2015

Citation: Shintani H (2015) Inactivation of Bacterial Spore, Endotoxin, Lipid A, and Abnormal Prion by Gas Plasma Exposure. Pharm Anal Acta 6: e173. doi:10.4172/2153-2435.1000e173

Copyright: ( 2015 Genel S, et al. This is an open-access article distributed under the terms of the Creative Commons Attribution License, which permits unrestricted use, distribution, and reproduction in any medium, provided the original author and source are credited. 


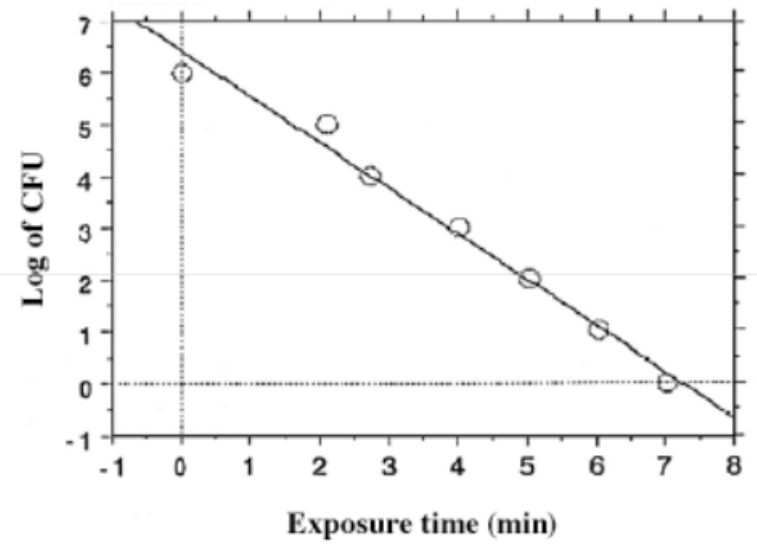

Figure 2: Straight survival curve when used clump-free biological indicator.

plasma sterilization against microorganisms or biomolecules has been extensively studied. According to our recent studies, the major factors causing sterilization by nitrogen gas plasma exposure was not well clarified and defined. In this editorial we present the basic principles of atmospheric and low pressure gas plasma sterilization and the active species produced and discuss their interactions with organic materials, pathogens (endotoxins, abnormal prion proteins), and bacterial spores $[1,3]$, showing how these factors could be used for sterilization and inactivation.
This editorial summarized the work carried out to date on lowpressure and atmospheric-pressure sterilization. The main mechanisms of plasma action on various biological pathogens have not well been identified and it has partly been shown that plasma treatment is a process for sterilization at low temperatures $[1,4]$. However, several unclear points still need to be clarified. For example, the sterilization mechanism is not completely clarified and the contribution factors of sterilization are not still identified. For medical application, it is required official approval beforehand, however in this status, approval may be quite difficult to obtain.

Apart from general process problems, the first issue is linked to the variability in efficiency of the sterilization mechanisms, which leads to difficulties in treating large loads with different shapes and sizes homogeneously. The second issue is linked to the matrix effect, which underlines the need to integrate plasma technology in a complete washing/cleaning process. The economics of the whole process including plasma discharge will need to be clarified, otherwise practical application of gas plasma cannot be utilized in the public in future.

\section{References}

1. Shintani H, Shimizu N, Imanishi $Y$, Sekiya T, Tamazawa K, et al. (2007) Inactivation of microorganisms and endotoxins by low temperature nitrogen gas plasma exposure. Biocontrol Science 12:131-143.

2. Shintani H, Sakudo A, Burke P, McDonnell G (2010) Gas plasma sterilization of microorfganisms and mechanisms of action (Review). Experimental Therpeutic Medicine 1: 731-738.

3. Shintani $H$ (2012) Inactivation of prion and endotoxins by nitrogen gas plasma exposure. Pharmaceut Anal Acta 3.

4. Shintani $H$ (2015) Major factors contributing nitrogen gas plasma sterilization. 